\title{
The Sidereal Center
}

\author{
Considerations Tending to Indicate That Canopus Occupies This Position
}

By. O. R. Walkey, F. R. A. S.

Тне Helium or B type, the so-called "Orion," stars are those which recent studies concur in placing at great distances from our system, while their brighter members, too, appear, according to Prof. Campbell (Lich Obs. Bul., 195) to lie as fully remote as do their fainter. This peculiarity, together with the rapid decrease, with diminishing magnitude, in the numbers of this class, argues a great average luminosity, coupled with grea distance. Further, these stars are found by Profs Campbell and Boss (Lick Obs. Bul., 195; Astron. Journ. $620,635-6)$ to be virtually free from that preferential motion, or star-streaming, which seems to be characteristic of the other spectral classes. These stars thus peculiarly invite treatment as of those most truly re peculiarly invite treatment as of those most truly re
presenting the visible universe, of which they may not unfairly be regarded as the framework.

In a recent study by the writer (Mon. Not. R.A.S May, 1914) of the distribution of the several spectra classes-particularly of type B-with reference to the galaxy, the relative preponderances of these stars $\left(\mathrm{Oe}^{-}\right.$ to B5) in galactic latitude and longitude respectively to B5) in galactic latitude and longitude respectively places the apparent center of their system on the 230 degree galactic meridian-or of "galongitude," reckoned
from the intersection of the galactic and celestial equators in Aquila-and between 20 degrees and 30 degrees south in galactic latitude, or of "galatitude." This, in other words, is the direction from wbich our solar system appears to lie eccentric within the lie verse defined by the helium verse defined by the helium stars. The relatively small number (706) of these stars involved (as given in $\mathrm{Har}$ vard Annals. Vol. 56, pt. 2) is largely compensated by the generally remote and therefore comprehensive distribution just referred to of this type's brighter mem

bers, of which this number is largely composed, and bers, of which this number is largely composed, and
includes them all. The sidereal center probably lies. therefore, on the line just named, or between $\alpha 6$ hours 0 minutes, $\delta-55$ degrees, and a 6 hours 55 minutes, $\delta-52$ degrees. The same studies with the work of Kapteyn, Boss, and Campbell further indicate the distance of this center as being in the neighborhood of 400 light-years, or 140 "secpars," to adopt under this name the more useful unit of stellar distance corresponding to 1.0 second parallax. ${ }^{1}$

The association of a particular star with the sidereal center would, if established, give coherence to the several lines of study now proceeding in stellar motion and distribution, and enable us to realize more clearly their true significance. Accepting the frosition line defined, it will be found to lie nearly symmetrically across the splendid star Canopus, and the only star within the prescribed limits of which we have any definite knowledge. This star appears to be the greatest sun of which we know anything, and is referred to by Prof. of which we know anything, and is referred to by Prof
See as an example of his hypothesis of condensed starSee as an example of his hypothesis of condensed star-
clusters In view of this remarkable coincidence the further study of this star assumes a peculiar interes as pertinent to the question of a sideral center.

First, as to the distance of Canopus: the late Sir David Gill found its parallax and proper motion to be insensible with regard to four 8th-magnitude comparison stars, of which the average absolute parallax, according to Prof. Kapteyn's table -Gron. Pub., No. 24) is 0.0065 seconds. Considering, however, the uncertain applica tion of averages to individuals, further evidence is detion of averages to individuals, further evidence is de-
sirable in support or otherwise of this value, and is best afforded by a study of the cross and radial motions i relation to the solar motion in space. The speed of solar motion and its apex appears to vary slightly, according to the spectral class of the stars to which they are referred. Here, as for their general fixity, the helium stars are the chosen reference for the solar position, so in consistence to them also should the direction and speed of solar motion be referred. Prof. Boss (Astron. Journ., $623-4$ ) has derived the solar apex relative to 490 of these stars alone, the mean of his two solutions placing the apex in $\alpha 18$ hours 17 minutes, $\delta+35$ degrees 0.4 (as

* From Knowledge.

1 The name here adopted is the proposed "parsec," with the syllables reversed to meet the objection lately raised to its im-
plication (by English usage of final syllables) of time or pure arc plication (by English
rather than distance.

suming a solar velocity of 20 kilometers a second); while Kapteyn, Campbell, and Stroobant (assuming apices derived from all spectral types, and but little removed from Boss) derive from the B stars solar velocities rang-
ing from 20.7 to 23.3 kilometers per second; the mean of these (weighted according to the number of stars used) is 21.5 kilometers per second, and is here adopted with Boss Apex, of which the antapex lies therefore in $\alpha 6$ hours 17 minutes, $\delta-35$ degrees 0.4 , a point found to lie 21 degrees 9 minutes south in galatitude (referred to Newcomb's mean north galactic pole in $\alpha 12$ hours 48 minutes, $\delta+27$ degrees) The antapex further lies 17 degrees utes, $\delta+27$ degrees) The antapex further lies 17 degrees
15 minutes northward from Canopus, the speed of apparent or parallactic drift of which (due to the solar motion) becomes 6.37 kilometers per second towards this antapex. The corresponding radial component (recessional) set up in Canopus is 20.53 kilometers per second, a figure which almost exactly coincides with the observed velocity of Canopus, 20.6 kilometers per second in recession. This indicates Canopus to be stationary with regard to the universe defined by the B type

The annual proper (cross-) motion of Canopus, ac cording to Bos ("Prelim. Gen. Cat.") is 0.0184 seconds

. 
is found to be 0.0505 seconds (clockwise) as against 0.0344 seconds (outward) mean radial motion. Of the tangential components only two are negative and one zero, while their stated mean represents 83 per cent of the mean whole motion. This, coupled with the fact that the negative radial components confine themselves that the negative radial components confine themselves to one side (west) of their primary, appears to afford
evidence of orbital motion enough to justify a closer study in weeding out any stars plainly extraneous to any presumable sub-Canopic system. An inspection of Fig. 289 will eliminate Nos. 10, 13, 17, and 22, as having larg proper motion, and apparently sharing the drift of the more rapid of Kapteyn's two-star streams (that toward $\alpha 90$ degree, $\delta-12$ degrees), their large P.M. arguing a common greater proximity to our system. On thi score No. 21, with 0.15 seconds in the opposite direction, should also be excluded. ${ }^{5}$

The relative motions of the remaining 18 stars, as of those at presumably the same order of distance, will remain unaffected by the component of solar motion, and, as possible satellites to Canopus, present the following quantities, which have been derived as simple means as sufficiently approximate, considering the inherent uncertainties of our data:

$$
\text { CANOPIC System. }
$$

Mean distance from Canopus.

Mean tangential component (clock-
wise)......................... 0.0341 seconds
Mean radial component (outward).... R 0.0042 seconds

Mean inclination to radius-vector ....

$$
\frac{-1 T}{R}=683 \mathrm{deg} .0 .0
$$

Minimum mass (times the Sun)...... 794,000

The angle $\theta$ expresses in its approach to 90 degrees that towards the condition of satellites symmetrically placed in a common orbital plane, or of circular motion in a plane normal to the right line. This latter case if true, would represent the minimum mass $\mathrm{M}=\mathrm{DT}$ when at a distance of 150 scepars, D represents 1,200 , 000 astronomical units, and T 15 miles a second, or 0.81 f 15 miles a second, or 0.8 of the earth's mean orbital
speed. This mass and that previously derived adepend previously derived adepend
alike on the adopted disalike on the adopted dis-
tance of Canopus, of which their mutual relation is consequently independent. Though necesarily provisional, the strikingt agreement after due allowance ment, after due allowance for coincidences al the two entirely indep mates of the minimum mass constitutes a somewhat forceful case for the reality of orbital motion for the faint star in the vicinity of Canopus. This of course, cannot be accepted as fact until we have the evidencze of such stars of the -52 degree to -55 degree belt in the southern vicinity of Canopus, as may reasonably lie at the same distance. The reduction of these motions is therefore highly desirable, as invested with a peculiar interest. There is in this connection one point to be observed, namely, that "forserved, namely, that "forward" orbital motions distributed inall planes around
their primary will appear "forward" or "retrograde" according as their stars lie on the near or far side of their primary. The settlement of the question thus awaits the development of appliances able to measure the radial velocities of these faint stars.

The actual mass of Canopus will be anything greate than the figure derived, according to the mean orbita inclination (i) to the line of sight. The most reasonable index as to this will follow from the assumption of random orbital inclination, whence integrating $\sin ^{3} i$ (by the inverse of which the mass varies) gives a mean value 0.589: this, divided into the minimum mass, gives $1,350,000$ times the Sun as the probable actual mass of Canopus.

A curious result which follows from this is that such 6Other stars, such as Nos. 1, 14, and 15, might further b omitted: such exclusion, however, is scarcely warranted in view
of the uncertainties of small P.M.'s, while their exclusion effect no appreciable change in the result ant quantitios

o The two stars of known P.M. in this region, \& Pictoris (4.8 magnitude) and $G$ Carinae (4.4 magnitude), have nearly equa and parallel motions (coun drift and considering too their brightness, and being only two in number, they supply no real criterion. a mass, acting at 150 secpars, would of itself produce in our Sun's motion a tangential component of 3.86 miles or 6.21 hilometers a second. When we consider that the actual crosswise component of solar motion is 6.37 kilometers per second, the striking agreement, which it seems hard to believe entirely coincident, impresses itself in confirmation of the foregoing hypothesis. Again, this agreement of the estimate with the fact supplies an indirect confirmation of the distance of Canopus, since this is ultimately a direct function of the velocity. The mass necessary at 150 secpars to produce the actual crosswise solar component would be $1,420,000$ Suns. The close agreement of these estimates implies that the combined attraction of the lesser suns anplies us is balanced in this respect-a state of affairs which, if continuous, would indicate for our Sun a highly eccentric hyperbolic orbit in a plane nearly perpendicular to the celestial, and having its "outer" focu distant 160 secpars in the direction $\alpha 6$ hours 14 minutes, $\delta-18$ degrees, 10 minutes (a degree west of $\beta$ Canis Majoris), towards which point, therefore, the solar antapex would imperceptibly shift with the lapse of time. As a matter of curiosity - for it assumes continued absence of disturbing forces-the elements of the hyperbola derived are given as follows: 8.52

Angle between asymptotes. $166 \frac{1}{2}$ degrees

Direct axis.......... $\quad 10.9$ secpars; inclined 13

Conjugate axis

degrees to galactio plane

degrees to galactic plane

Parameter

780 secpars; inclined 23 degrees to galactic plane

"Perihelial" distance. .

Epoch since "periastral" passage.. $219 \times 10^{12}$ years

Needless to say, the final item is imaginary of the

(a) The objectives ( $\alpha 90$ degrees, $\delta-12$ degrees and
263 degrees, $\delta-60$ degrees) of Kapteyn's two starstreams (assuming their reality) lie on either side of their respective speeds, and in nearly the same apparent south galatitude as Canopus.

(b) The solar antapex lies in nearly the same apparent south galatitude.

It will, of course, be clearly understood that none of the foregoing indications are set forth as in any way proving the central position of Canopus, the indication in some cases being within its own limit of error. They nevertheless point consistently in the same direction, though mutually independent, so that, however slender may be each cord in itself, yet, collectively, they assume that comparative strength which the cable bears to its component strands. As a chain of mere coincidences in a common direction, the foregoing indications would be less remarkable than the fact they demonstrate.

Finally, the central position of Canopus may, if established, be used to determine the galactic distances in terms of the Sun-Canopus line, which would thus serve for the sidereal system the purpose served by our astronomical unit (Earth-Sun line) for the solar system. The table summarizes the resultant distances

\begin{tabular}{|c|c|c|c|}
\hline Quantity. & Unital. & Secpars. & $\begin{array}{l}\text { Light } \\
\text { years. }\end{array}$ \\
\hline 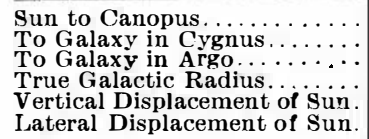 & $\begin{array}{c}1.00 \\
12.98 \\
14.80 \\
13.88 \\
0.422 \\
0.906\end{array}$ & $\begin{array}{r}150 \\
1,950 \\
2,220 \\
2,080 \\
63,3 \\
136\end{array}$ & $\begin{array}{r}489 \\
6,330 \\
7,220 \\
6,780 \\
206 \\
442\end{array}$ \\
\hline
\end{tabular}
relative to the unit line, and absolutely in secpars

and light-years. The quantities follow from the apparent southward deviation 1.75 degrees, derived by the late Prof. Newcomb for the main galactic stream in connection with the apparent south galatitude 25.1 degrees of Canopus, relative to the plane having its N. pole in $\alpha 192$ degrees,

Our eccentricity within the galaxy is therefore vertically $1 / 33$ and horizontally $1 / 15$ of the galactic radius, while the figure derived for this radius, corresponding to a parallax of 0.0005 seconds, follows the trend of recent indications by other methods.

While considering the distance of the galaxy, an independent and rough index of this is supplied from the average actual luminosity in terms of our Sun of the Type II (F, G, K) stars, of which the class Faith's integrated spectrum ( $A p$. Journ., volume 36 , page 362 ) indicates the galaxy mainly to consist. According to
all the recent studies embodied in Prof. Russell's bodied in Prof. Russell's gram, these stars average at 0.8 "sunpower," and, taking these to be represented by the average galactic star of apparent magnitude 17, their mean parallax becomes 0.00045 seconds, giving a distance of 2,230 secpars, or 7,250 light-years, which strikingly confirms

present creation being then existent! The angular divergence of the asymptotes indicates (in its supplement) that the ultimate deviation of the solar path due to Canopus is $13 \frac{1}{2}$ degrees from the straight line.

The several indications in favor of the present hypothesis may now be summarized as follows:

(i) Canopus occupies the approximate position derived for the center of the stellar system, as defined by the remote helium stars.

(ii) Its distance is of the same order as that indicated r this helium star center.

(iii) Canopus appears to be stationary with reference o these virtually "fixed" helium stars.

(iv) Its predominant luminosity and mass are in haracter with their suggested significance.

(v) The relative motions of the faint stars, so far examined, in the vicinity of Canopus indicate an orbita motion confirming the independently derived mass.

(vi) The component of solar motion tangential to Canopus indicates the existence of such a mass at the given distance.

Two other features, which may prove relevant, are: the present estimate, and consequently the suggested Canopic hypothesis on which it rests.

There is, to conclude, one factor not to be ignored, and which, though at first sight irrelevant, yet, if established. should be decisive on the question of visible galactic distances, and that is the date of Creation. If the true interpretation of the period named in the inspired account (Genesis i) be six literal days-for which the Hebrew evidence seems strong - and if, again (though the evidence is not so definite), these days apply to the (Concluded on page 394.)

${ }^{7}$ The galactic meridian of Canopus bisects the remarkable assemblage of helium stars in a belt 10 degrees wide between 200 dequarter of the present count for the whole sky.

quarter of the present count for the whole sky.
8 This pole seems to give the simplest representation of the truth, being the mean of Newcomb's reductions: Newcomb's pole for the main stream alone lies in $a 192$ degrees 0.8 minutes, $\delta+27$ degrees 0.2 , the deviation of which, however, from that adopted here leaves the mean southward dip unaffected.

9
This would seem to dispose of Prof. See's exceptional estimata tion that the galactic stars are type $B$ of nearly one thousand "sun-power." 